\title{
Unexpected spatial intensity distributions and onset timing of solar electron events observed by closely spaced STEREO spacecraft
}

\author{
A. Klassen ${ }^{1}$, N. Dresing ${ }^{1}$, R. Gómez-Herrero ${ }^{2}$, B. Heber ${ }^{1}$, and R. Müller-Mellin ${ }^{1}$ \\ ${ }^{1}$ Institut für Experimentelle und Angewandte Physik, Universität Kiel, 24118 Kiel, Germany \\ e-mail: klassen@physik.uni-kiel.de \\ 2 SRG, Universidad de Alcalá, Dpto. de Física y Matemáticas, 28871 Alcalá de Henares, Spain \\ Received 18 April 2016 / Accepted 18 May 2016
}

\begin{abstract}
We present multi-spacecraft observations of four solar electron events using measurements from the Solar Electron Proton Telescope (SEPT) and the Electron Proton Helium INstrument (EPHIN) on board the STEREO and SOHO spacecraft, respectively, occurring between 11 October 2013 and 1 August 2014, during the approaching superior conjunction period of the two STEREO spacecraft. At this time the longitudinal separation angle between STEREO-A (STA) and STEREO-B (STB) was less than $72^{\circ}$. The parent particle sources (flares) of the four investigated events were situated close to, in between, or to the west of the STEREO's magnetic footpoints. The STEREO measurements revealed a strong difference in electron peak intensities (factor $\leq 12$ ) showing unexpected intensity distributions at $1 \mathrm{AU}$, although the two spacecraft had nominally nearly the same angular magnetic footpoint separation from the flaring active region (AR) or their magnetic footpoints were both situated eastwards from the parent particle source. Furthermore, the events detected by the two STEREO imply a strongly unexpected onset timing with respect to each other: the spacecraft magnetically best connected to the flare detected a later arrival of electrons than the other one. This leads us to suggest the concept of a rippled peak intensity distribution at $1 \mathrm{AU}$ formed by narrow peaks (fingers) superposed on a quasi-uniform Gaussian distribution. Additionally, two of the four investigated solar energetic particle (SEP) events show a so-called circumsolar distribution and their characteristics make it plausible to suggest a two-component particle injection scenario forming an unusual, non-uniform intensity distribution at $1 \mathrm{AU}$.
\end{abstract}

Key words. Sun: flares - Sun: particle emission - acceleration of particles

\section{Introduction and motivation}

Previous single spacecraft $(\mathrm{s} / \mathrm{c})$ observations and propagation models of solar energetic particle (SEP) events suggest a relatively simple and uniform particle intensity distribution not only at 1 AU but also close to the Sun (e.g., Kallenrode 1993; Strauss \& Fichtner 2015). A symmetric Gaussian intensity distribution is assumed with a maximum on field lines connecting to the source (flare/CME) with a strong intensity decrease perpendicular to the magnetic field or the nominal Parker spiral (e.g., Dröge et al. 2010). However, the real spatial distribution of particles close to the Sun is not known, and the injection distribution is assumed as a point-like or a spatially distributed source (e.g., Dröge et al. 2010).

Recent studies of so-called widespread or circumsolar SEP events (e.g., Dresing et al. 2012, 2014; Wiedenbeck et al. 2013; Lario et al. 2013; Gómez-Herrero et al. 2015) using multispacecraft observations by widely separated STEREOs and nearEarth $\mathrm{s} / \mathrm{c}$ found out that the particle distribution may be very broad up to 360 degrees around the Sun at 1 AU. For the majority of the events the intensity distribution shows a Gaussianlike shape. Nevertheless, the first detection of a SEP spike event at both closely spaced STEREO s/c (Klassen et al. 2015) and a small part of all widespread events reveal strong deviations from a simple-uniform Gaussian intensity distribution at 1 AU (e.g., Wibberenz \& Cane 2006; Dresing et al. 2012, 2014; Lario et al. 2013; Dröge et al. 2014; Richardson et al. 2014; Gómez-Herrero et al. 2015).

Since late 2013 through 2014 the two STEREO s/c approached their superior solar conjunctions (occurring in
February for STEREO-A (STA) and in May 2015 for STEREO-B (STB)) and the separation angle between both s/c was less than $72^{\circ}$. This unique constellation gives us an opportunity to study more precisely the particle distribution at $1 \mathrm{AU}$, especially when the parent particle source (e.g. the flaring active region (AR)) is located not far away from the magnetic footpoints of the two $\mathrm{s} / \mathrm{c}$ or is even located between them. Such a closely spaced s/c constellation makes it possible to investigate how uniform the SEP intensity distribution is at $1 \mathrm{AU}$ when it is examined over relatively narrow angular intervals. In a recent study Klassen et al. (2015) found hints towards a non-symmetric and a non-uniform intensity distribution of electrons with respect to the nominal Parker spiral at $1 \mathrm{AU}$.

We selected four solar electron events detected with the Solar Electron and Proton Telescopes (SEPT) on board the two STEREO s/c. Two of these events have also been detected by the Electron Proton Helium INstrument (EPHIN) on board SOHO. We present multi-spacecraft electron intensity and anisotropy observations at $1 \mathrm{AU}$, and we determine the particle onset delay between the two STEREO s/c as well as the delays with respect to the associated type III radio bursts. All presented events reveal an unconventional and unexpected behavior in the spatial intensity distribution and onset timing.

\section{Instrumentation}

The particle measurements are provided by identical SEPT on board the two STEREO s/c. "SEPT consists of two dual doubleended magnet/foil particle telescopes, which cleanly separate 
and measure electrons in the energy range from $30-400 \mathrm{keV}$ and protons from 60-7000 keV. Anisotropy information on a non-spinning $\mathrm{s} / \mathrm{c}$ is provided by the two separate telescopes: SEPT-E looking in the ecliptic plane along the Parker spiral magnetic field both towards and away from the Sun, and SEPT-NS looking vertical to the ecliptic plane towards North and South" (Müller-Mellin et al. 2008). The EPHIN instrument on board the non-spinning SOHO s/c measures amongst others electrons in the energy range $0.25-10.4 \mathrm{MeV}$ (Müller-Mellin et al. 1995). In addition, we use measurements from High Energy Telescope (HET; von Rosenvinge et al. 2008), Plasma and Suprathermal Ion Composition (PLASTIC) solar wind data (Galvin et al. 2008), the STEREO radio and plasma Wave (SWAVES) radio emission spectra (Bougeret et al. 2008), Extrem Ultra Violet Imager (EUVI; Howard et al. 2008), and Magnetometer (MAG) data (Acuña et al. 2008) from the two STEREO s/c.

\section{Observations}

From a number of SEP events detected at the two STEREO s/c since October 2013 we selected four events representing a specific constellation of flare locations relative to the $\mathrm{s} / \mathrm{c}$ nominal magnetic Parker spiral footpoints. The nominal Parker spiral and the nominal magnetic footpoints at the solar wind source surface are calculated using the measured solar wind speeds during the onset of the SEP event. During this period the orbital longitudinal separation angle between STA and STB decreases from $72^{\circ}$ on 11 October 2013 to $34^{\circ}$ on 1 August 2014. We selected SEP events with three different constellations:

1. The flare was situated between the nominal magnetic footpoints of the two STEREO s/c (event on 11 October 2013, Figs. 1 and 2);

2. The flare was situated to the west of both s/c footpoints $(25$ February 2014, Figs. 5 and 7);

3. The flare was situated very close to both $\mathrm{s} / \mathrm{c}$ footpoints (two events on 1 August 2014, Figs. 10 and 11).

It is important to mention that the two selected events on $11 \mathrm{Oc}$ tober 2013 (Sect. 3.1) and on 25 February 2014 (Sect. 3.2) represent so-called widespread or circumsolar particle events (e.g., Dresing et al. 2012; Gómez-Herrero et al. 2015). Both events are detected at all three widely separated locations of STA, STB, and SOHO and show a very broad longitudinal particle distribution.

\subsection{The 11 October 2013 SEP event}

The widespread SEP event on 11 October 2013 was detected at STA, STB and SOHO in a broad energy range from a few tens of $\mathrm{keV}$ up to $\geq 1 \mathrm{MeV}$ for electrons and in the range of $\leq 100 \mathrm{MeV}$ for protons. The location of the STEREO and SOHO $\mathrm{s} / \mathrm{c}$ is shown in the upper right panel of Fig. 1. The event was associated with an M1.5 X-ray flare which occurred $\sim 10^{\circ}$ behind the east solar limb (as seen from Earth). The flare was associated with a full-halo (1200 $\mathrm{km} \mathrm{s}^{-1}$ ) coronal mass ejection (CME), a dome-shaped circular EIT-wave ( speed $\geq 710 \mathrm{~km} \mathrm{~s}^{-1}$ ), a coronal type II radio burst starting at $0711 \mathrm{UT}$ at $270 \mathrm{MHz}$ followed by an IP type II radio burst observed by IZMIRAN and SWAVES, respectively.

Additionally, the flare was accompanied by an interplanetary (IP) type III radio burst at 0712-0725 UT, starting one minute after the type II burst onset. No type III bursts associated with the impulsive flare stage were observed. Furthermore, the SWAVES instrument at STA detected locally generated Langmuir waves,

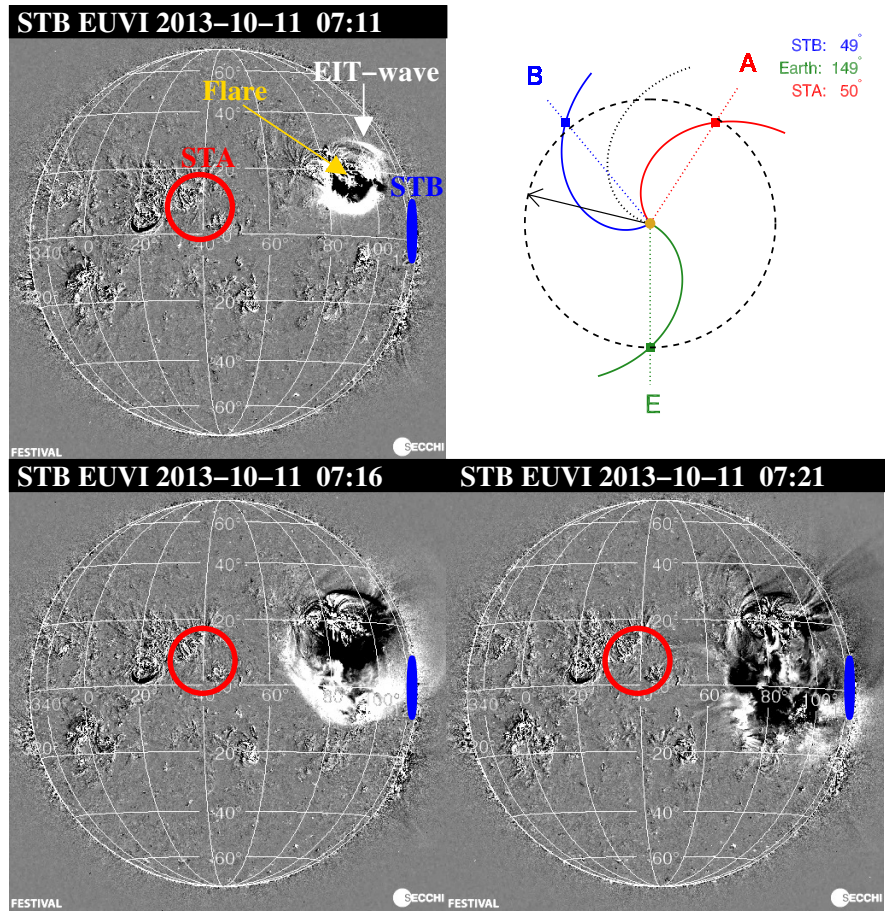

Fig. 1. STEREO-B EUVI $195 \AA$ A difference images showing the flare location and the evolution of the circular EIT-wave relative to the nominal magnetic footpoints of STA (red) and STB (blue) during the SEP event on 11 October 2013. Top right: longitudinal positions of STA, STB, and Earth/SOHO and the nominal Parker spirals connecting them to the Sun. The arrow indicates the location of the parent flare, the source of the SEP event. The angular separations between the s/c magnetic footpoints and the flare position are inserted in the upper right corner.

indicating the arrival of low-energy electrons generating the type III radio emission (not shown). Langmuir waves at STB and at the Wind s/c were not detected. From the STB point of view the flare occurred near N20W40 (Fig. 1), and for STA the flare was $\sim 20^{\circ}$ behind the west limb. Figure 1 (top right) presents the locations of the parent flare/AR, STA, STB, SOHO, and the nominal Parker magnetic field spirals connecting the $\mathrm{s} / \mathrm{c}$ to the Sun, corresponding to the solar wind speeds measured during the electron onset.

Figure 2 shows in the upper panel the time profile of 55 to $65 \mathrm{keV}$ electrons together with the 250-700 keV measurements by EPHIN at L1 from 10 October to 17 October 2014. Unfortunately, because of higher background, ACE at L1 did not detect this event at energies 55-105 keV. The three lower panels show an expanded view including pitch angle (PA) dependent intensity variations. Both STEREO s/c detected a prompt electron increase with a clear velocity dispersion (not shown) followed by a long decay in a broad energy range from tens of $\mathrm{keV}$ up to $1 \mathrm{MeV}$.

Figure 2 (bottom) presents the pitch angle distributions (PADs) as measured at both STA and STB s/c. During the event the STA and STB pitch angle coverage was quite good and the data show clear anisotropic electron fluxes from the event onset and lasting for several hours. Admittedly there are limitations for the pitch angle coverage of the SEPT instrument since it has only four viewing directions. Electron anisotropies at STA and STB show flux increases at the PA closer to $0^{\circ}$ and to $180^{\circ}$, respectively, which corresponds to particles propagating from the direction of the Sun but along opposite polarities. These polarities correspond to the open magnetic field 

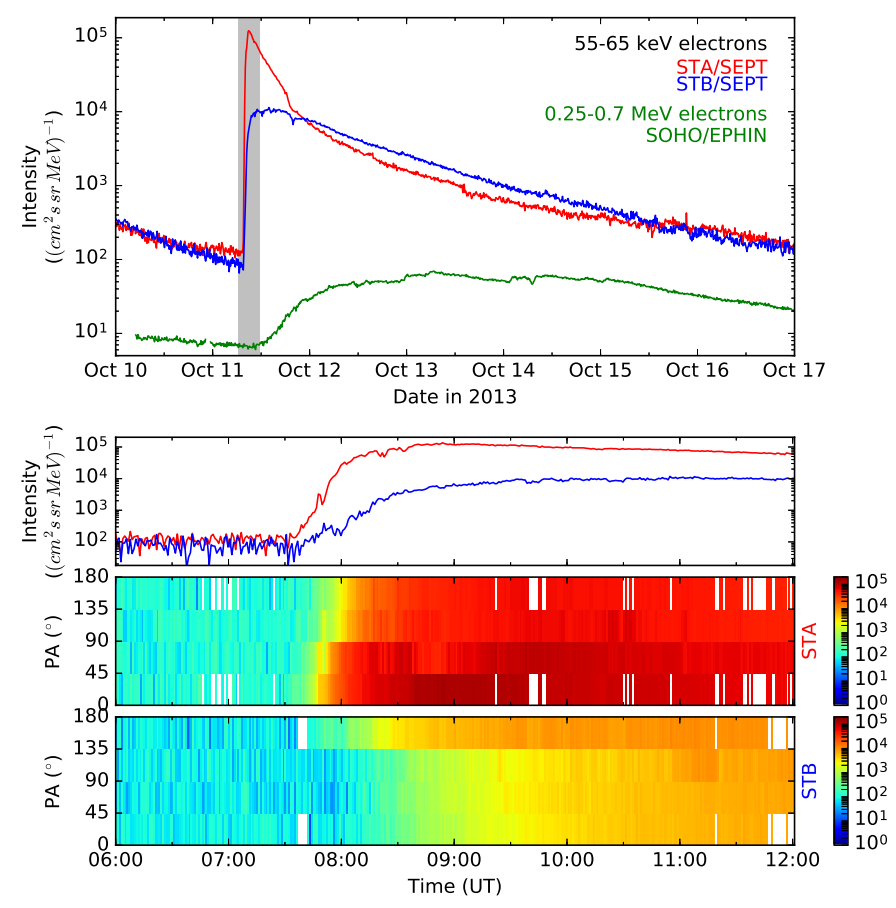

Fig. 2. Electron event on 11 October 2013. Top: intensity time profiles detected at STA (red) and STB (blue) showing distinct differences in peak intensities and in the onset times. For comparison the intensity profile at SOHO is shown in green. Bottom: enlarged time profiles and intensity pitch-angle distributions measured by STA and STB (shaded interval on the top).

distribution presented in Fig. 3 (red denotes negative polarity, green positive polarity). This suggests that the particles were injected into or along different magnetic sectors from the flare/AR site, which lies in projection close to the global heliospheric current sheet as indicated by the white line. This particle event at SOHO was more than two orders of magnitude weaker than at STA (at the same energy of $0.7 \mathrm{MeV}$, as shown in Fig. 15 ) and was delayed by about $200 \mathrm{~min}$ with respect to the other s/c.

Figure 3 presents a synoptic global oscillation network group (GONG) map with open magnetic field lines connecting to the ecliptic plane calculated using the potential field source surface (PFSS) model, overplotted at the solar magnetogram taken on 11 October 2013. The back-mapped locations of the nominal magnetic footpoint of all s/c are displayed as crosses and circles in red, blue, and green for STA, STB, and SOHO, respectively. The circles denote $\mathrm{a} \pm 10^{\circ}$ uncertainty and the location of the parent flare is marked by a gold diamond.

Although the flare was located nearly in between of the nominal connecting footpoints of STA and STB with an angular separation to the flare of $50^{\circ}$ for STA and $49^{\circ}$ for STB (see Table 1), the measured peak intensities at STA and STB differ strongly and unexpectedly by a factor of $\sim 12$ (Fig. 2). Furthermore, the electron onset at STB is delayed with respect to STA by $13 \mathrm{~min}$, whereas the electron onsets with respect to the associated type III radio bursts are delayed by about 19 and 8 min for STB and STA, respectively (see Sect. 3.4 for the delay calculations).

\subsubsection{In situ plasma and magnetic field observations and IP conditions during the 11 October 2013 event}

Figure 4 presents an overview of in situ plasma and magnetic field data as observed by STA and STB from 10 October to

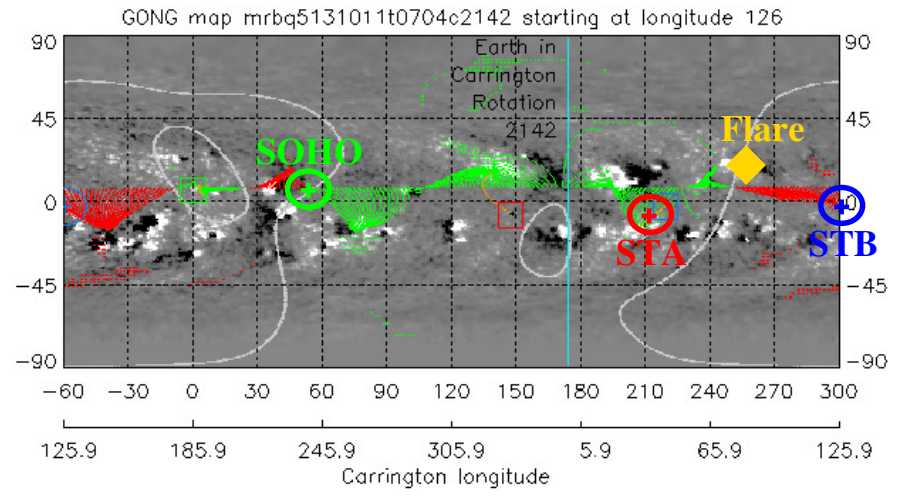

Fig. 3. Synoptic magnetic field map from $\mathrm{GONG}^{1}$ showing the PFSS ${ }^{1}$ open magnetic field lines connecting to the ecliptic plane on 11 October 2013. S/c magnetic nominal footpoints are indicated by circles. STA is shown in red, SOHO in green, STB in blue. The location of the associated flaring AR is indicated by a gold diamond.

17 October 2013. The panels show from top to bottom the solar wind proton speed, density, temperature, magnetic field magnitude, its latitudinal angle and its azimuthal angle in the RTN coordinate system. During the SEP event, indicated by a solid vertical line, and during the whole time interval the IP environment implies relatively quiet conditions when both $\mathrm{s} / \mathrm{c}$ were embedded in slow solar wind $\left(V \mathrm{sw}<380 \mathrm{~km} \mathrm{~s}^{-1}\right)$ and in relatively quiet magnetic fields. No interplanetary coronal mass ejections (ICMEs) and co-rotating interaction regions (CIRs) were observed during the whole period.

According to the L.K. Jian shock list ${ }^{2}$ only two weak IP shocks in the slow solar wind speed were detected at STB on 12 October (at 1709 UT) and 15 October (at 1552 UT) marked by dashed lines in Fig. 4. It is not clear whether these shocks were in fact associated with the CME/ICME launched during the SEP related flare, and were indeed en route between the Sun and STEREO and thus were able to modify the IP magnetic field and the nominal propagating path of electrons.

The second shock on 15 October was presumably associated with the SEP parent flare/CME on 11 October. In this case it has no influence on the particle propagation. The first shock on 12 October could be in fact situated between the Sun and the $\mathrm{s} / \mathrm{c}$ during the event. However, its influence on the particle propagation and on the shape of the Parker spiral was rather small because it appears in slow solar wind and without an ICME signature behind the shock.

During the three days prior to the SEP event only slow CMEs $\left(<520 \mathrm{~km} \mathrm{~s}^{-1}\right)$ were observed with Large Angle and Spectrometric Coronagraph (LASCO) ${ }^{3}$ confirming a lack of magnetic disturbances in the IP medium as shown in Fig. 4. All these facts together support the suggestion that during the SEP event the nominal Parker magnetic field structure was not strongly modified by the observed transients and that the estimated nominal STEREO magnetic footpoint positions shown in Fig. 3 corresponded to the actual positions connecting the source surface with both s/c.

In summary: on the one hand, the electron event on 11 October 2013 shows a clear velocity dispersion and anisotropic PADs at the two STEREO s/c implying optimal connections to the

\footnotetext{
1 GONG: Global Oscillation Network Group, PFSS: Potential Field Source Surface.

2 http://www-ssc.igpp.ucla.edu/ jlan/STERE0/Level3/

STEREO_Level3_Shock.pdf

3 http://cdaw.gsfc.nasa.gov/CME_list/index.html
} 
STEREO-A and STEREO-B Plasma and Magnetic Field Data

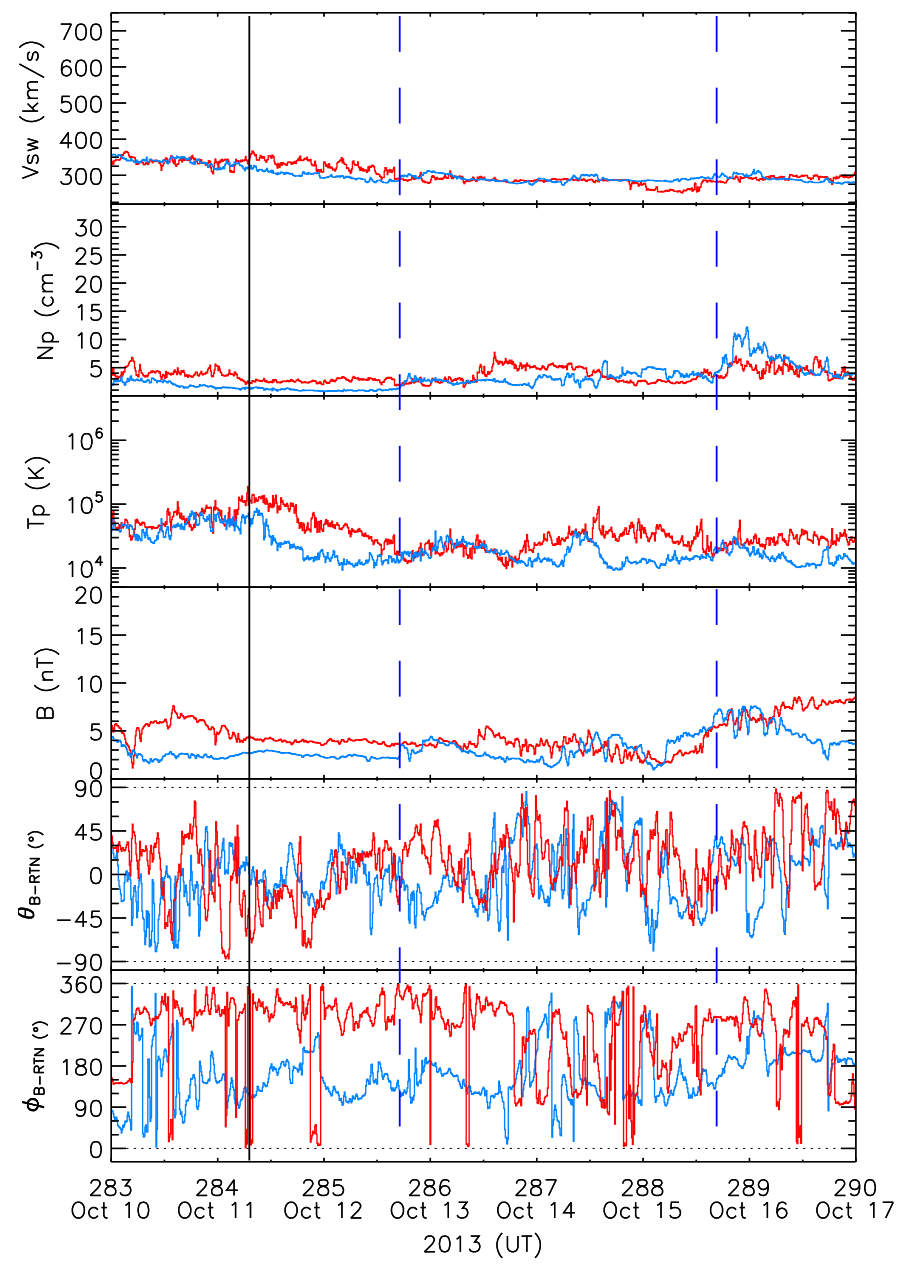

Fig. 4. Event on 11 October 2013. Solar wind and magnetic field measurements from 10 to 17 October 2013 (red: STA, blue: STB). From top to bottom: solar wind proton speed, proton density, proton temperature, magnetic field magnitude, magnetic field latitudinal, and longitudinal angles. The vertical solid line marks the SEP onset and the dashed lines mark IP shocks observed at STB.

parent particle source although the peak intensities at the two $\mathrm{s} / \mathrm{c}$ differ strongly by a factor of 12 . On the other hand, the s/c nominal angular magnetic footpoint separations to the flare were closely comparable by $\sim 50^{\circ}$ westward and $\sim 49^{\circ}$ eastward, for STA and STB, respectively. The Parker spirals connecting the $\mathrm{s} / \mathrm{c}$ to the Sun were probably not essentially modified because of the relatively quiet IP conditions. Consequently, the strong peak intensity difference might be explained assuming an expanded source with a strong non-uniform particle distribution below the source surface and in the IP medium. Another possibility might be a strongly non-radial magnetic field structure between the flare and the source surface.

\subsection{The 25 February 2014 SEP event}

The 25 February 2015 SEP event was associated with a X4.9/2B $\mathrm{X}$-ray/ $\mathrm{H}_{\alpha}$ flare (start 0039, maximum $0049 \mathrm{UT}$ ) appearing close to the solar east limb (Earth view) at coordinates S12E82. Viewed from STB it appears at the west limb (Fig. 5), and viewed from STA the associated flare occurs $40^{\circ}$ behind the west limb (Fig. 6).
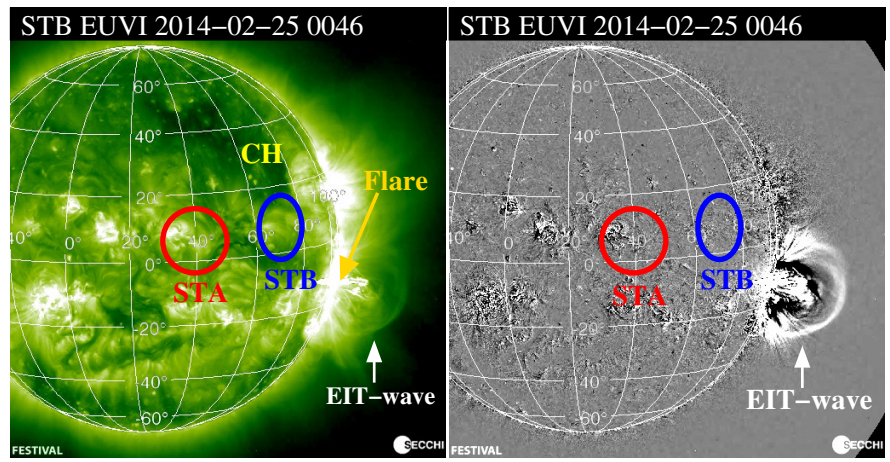

Fig. 5. STEREO-B EUVI $195 \AA$ direct (left) and difference images (right) showing the flare location, the dome-shaped EIT-wave, and the coronal hole $(\mathrm{CH})$ in relation to the nominal magnetic footpoints of STA (red) and STB (blue) during the SEP event on 25 February 2014.

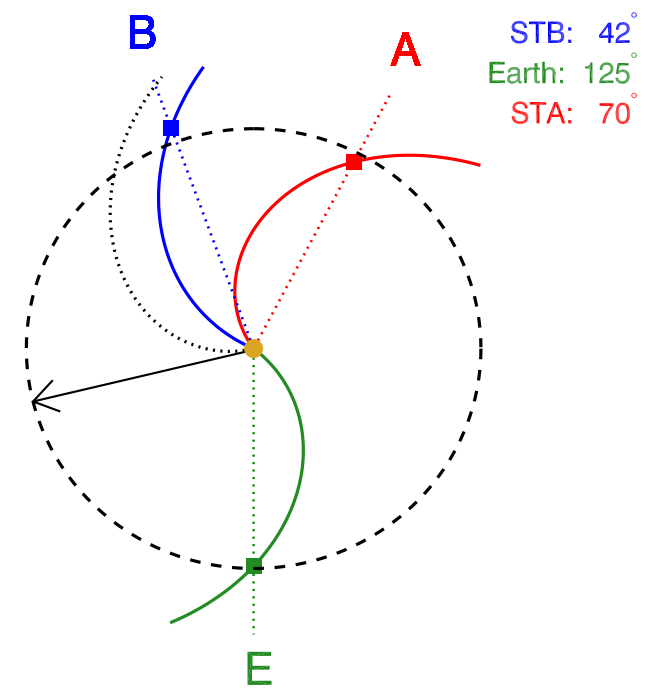

Fig. 6. Longitudinal positions of STA, STB, and Earth/SOHO and the nominal Parker spirals connecting them to the Sun. The black arrow indicates the location of the parent flare, the source of the SEP event on 25 February 2014. The angular separations between the s/c magnetic footpoints and the flare position are inserted in the upper right corner.

The flare was accompanied by IP type III and coronal-IP type II radio bursts starting simultaneously at 0145.5 UT, and by a fast asymmetric full-halo CME (speed $\leq 1780 \mathrm{~km} \mathrm{~s}^{-1}$ ) and by a dome-shaped EIT-wave as shown in Fig. 5. The nominal footpoints of STA and STB were situated definitely eastwards from the flare location.

Figure 7 shows the electron intensity time profiles from the two STEREO s/c in the energy range of 55-65 keV measured with the sunwards looking telescopes of SEPT together with the 250-700 keV measurements by EPHIN. Apart from the fact that STB shows an onset that is 13 min later than shown by STA, the shapes of both time profiles are similar to each other from onset on and during the following two hours when the intensity at STA reached its maximum. Afterwards the STA intensity profile decays gradually, while the STB intensity shows a slowly growing profile in the next $15 \mathrm{~h}$ which can be explained by an additional acceleration of the approaching IP shock.

Figure 7 (bottom) shows the SEPT/STA PAD with a clear moderate anisotropy from onset through the intensity maximum when the main part of particles streamed from the Sun at PA $180^{\circ}$. The PAD for STB is not available owing to a lack of magnetic field data. 

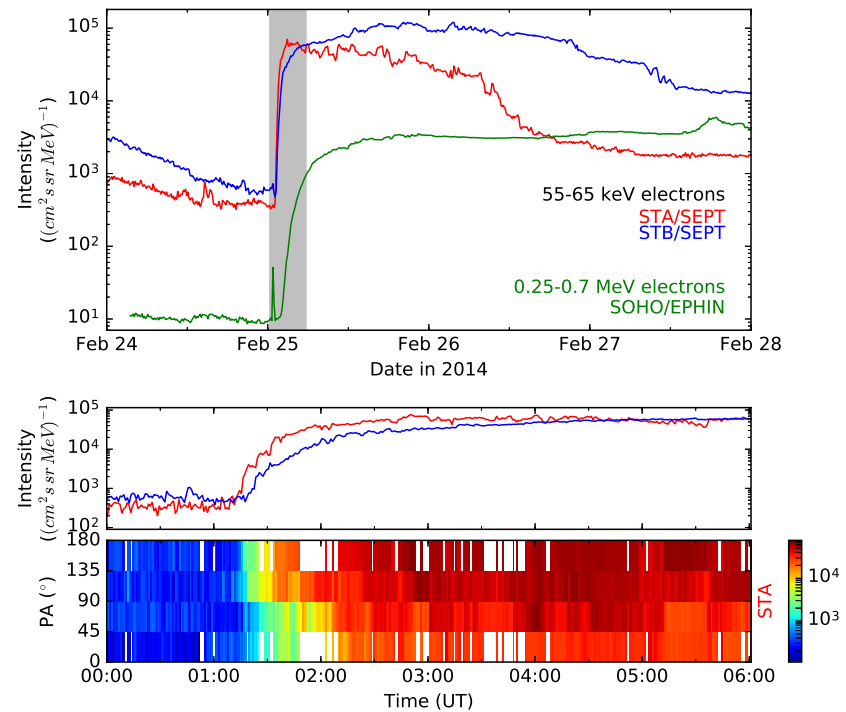

Fig. 7. Event on 25 February 2014. Top: electron time profiles detected at STA (red), STB (blue), and SOHO (green). STA shows an earlier onset and higher intensities at the beginning of the event than STB. The spike before the event onset in EPHIN data is due to the hard X-ray contamination. Bottom: enlarged interval (shaded period on the top) of time profiles at STA and STB. The PAD measured by STA shows a clear anisotropy from the event onset until 02:00 UT. PAD data for STB are not available because of a data gap in MAG data.

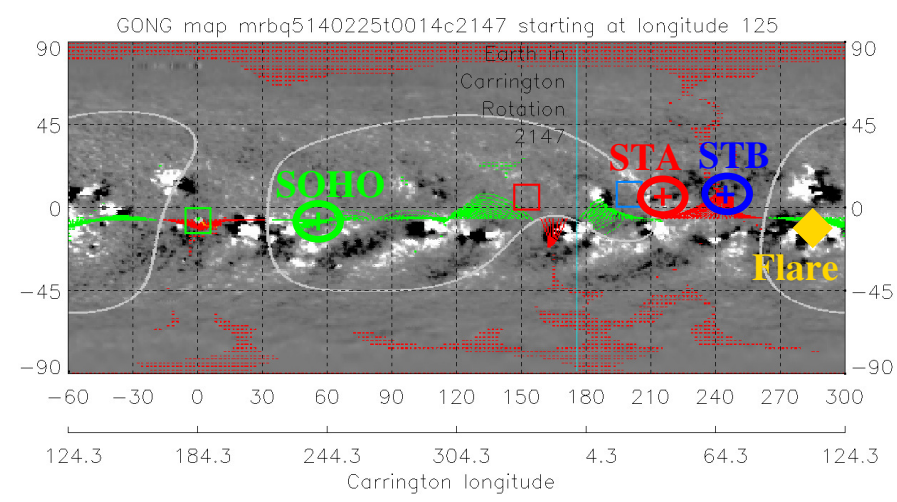

Fig. 8. Synoptic magnetic field map from GONG for the event on 25 February 2014. Description as in Fig. 3.

A synoptic magnetic field map from GONG showing the PFSS open magnetic field lines connecting to the ecliptic plane is presented in Fig. 8. S/c nominal footpoints are indicated by circles (STA in red, SOHO in green, STB in blue). The location of the flaring AR is marked by a diamond. The footpoints of both STEREO s/c are situated eastward from the flaring AR; STA is farther away than STB. Nevertheless, the particle onset at STA was 13 min earlier and the intensity during the first hours was higher and peaked at $0250 \mathrm{UT}$, clearly earlier than at STB as shown in Fig. 7. The onset delay with respect to type III bursts is 10, 24, and 78 min for STA, STB, and SOHO, respectively. This means - if we take only the STEREO observations - that the particles arrived and peaked first at the STA s/c, which was farther away (separation to the flare $70^{\circ}$ ), and then at the nominally best connected STB with a separation of $42^{\circ}$.

Figure 9 presents in situ plasma and magnetic field data measured by the two STEREO s/c from 24 February to 3 March. The onset of the SEP event is indicated by the solid line. It occurs during different interplanetary conditions for the two STEREO

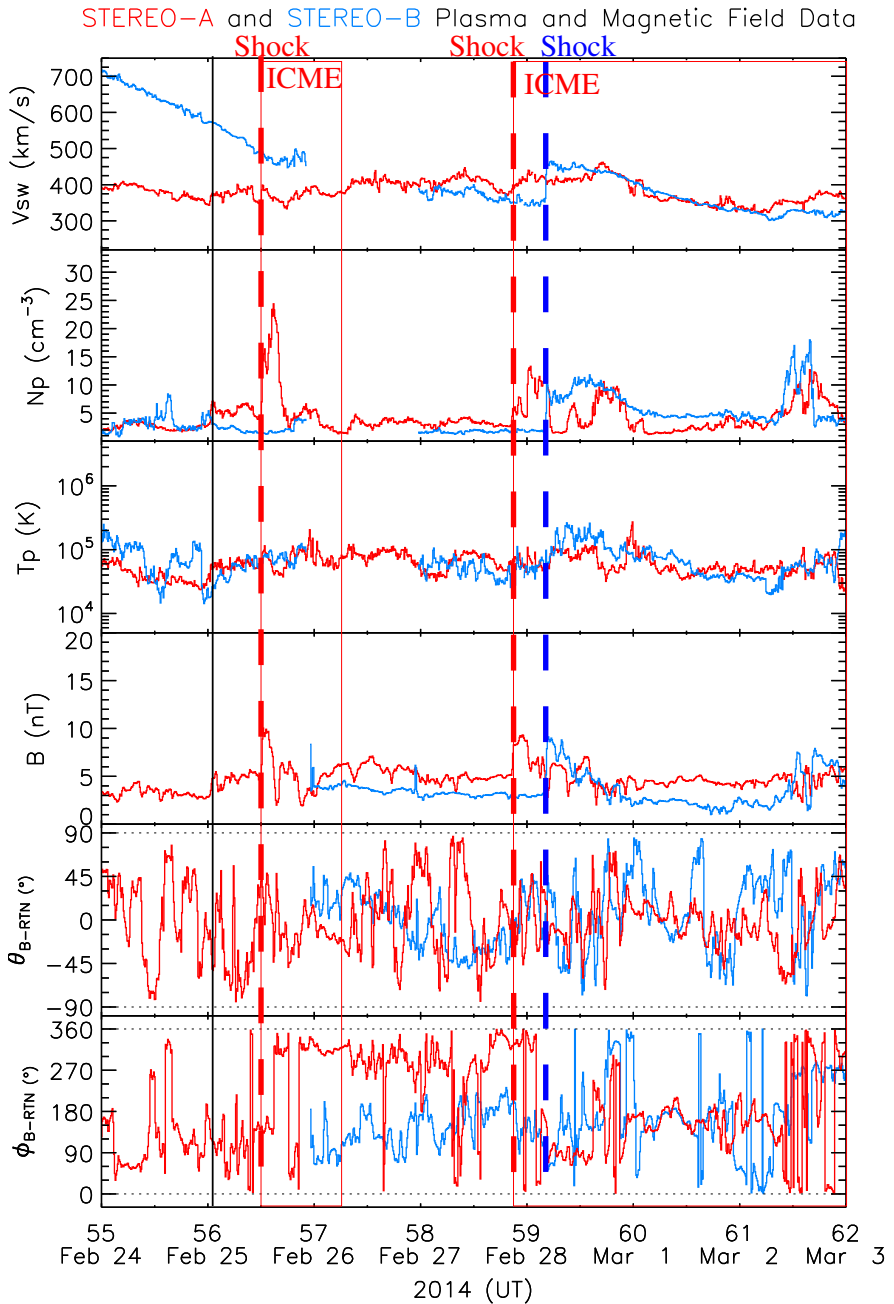

Fig. 9. Event on 25 February 2014. Solar wind and magnetic field data as in Fig. 4. SEP onset and IP shocks are indicated by solid and dashed vertical lines, respectively. Magnetic field data from 20 to 26 February and solar wind data from 25 to 27 February were not available.

s/c. During the SEP onset at 0109 UT STA was situated in a slow solar wind stream $\left(370 \mathrm{~km} \mathrm{~s}^{-1}\right)$ followed by a weak IP shock at 1216 UT and an ICME, while STB was situated inside a highspeed stream $\left(570 \mathrm{~km} \mathrm{~s}^{-1}\right)$. The high-speed stream very likely originated from the coronal hole $(\mathrm{CH})$ situated close to the STB footpoint as shown in Fig. 5. The ICME detected at STA and not at STB might change the IP magnetic field connecting STA with the Sun and might shift the nominal footpoint to the west, i.e. closer to the flare. On the other hand, because this ICME was not detected at STB its extent was obviously not large and the shift was likely not essential.

Although the SEP associated CME was not directed towards the Earth/STA/STB, it drove an IP shock that possible overtook all three s/c: SOHO at 1617 UT on 27 February, STA at 2046 UT on 27 February, and STB at 0423 UT 28 February. Consequently the shock angular width was at least $\geq 207^{\circ}$ because the separation angle between STA and SOHO/L1 was $207^{\circ}$ and the flare/CME was located in between. As far as we know from the literature it could be the broadest IP shock ever observed because, according to Cane (1996), the longitudinal extent of IP shocks at $1 \mathrm{AU}$ is at most $180^{\circ}$. On the other hand, there is an uncertainty of the shock origin at STA (Lario et al. 2016). 


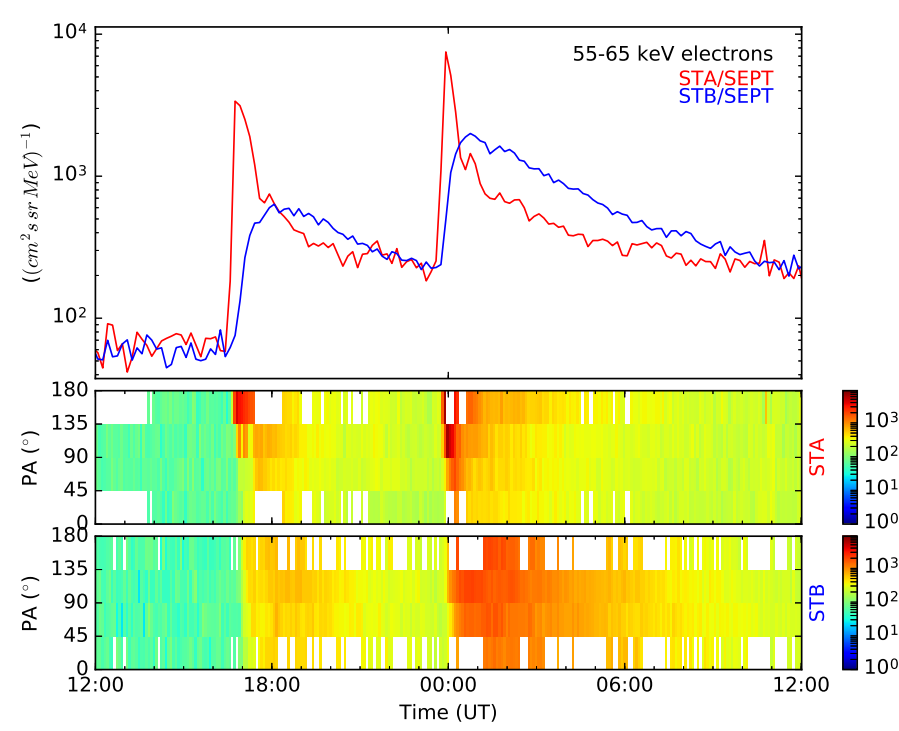

Fig. 10. Events on 1 August 2014. Electron intensity time profiles detected at STA (red) and STB (blue). During both events STA shows an earlier onset, higher intensity, and stronger anisotropy than STB.

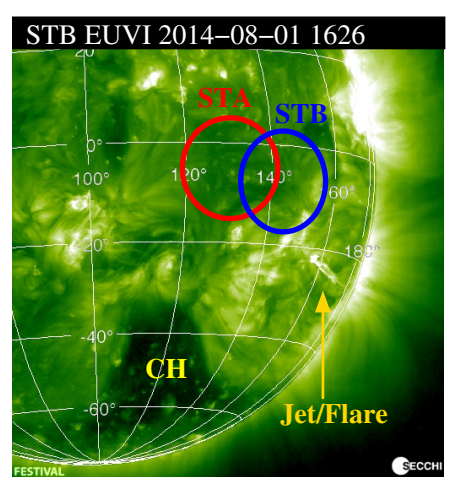

(a)

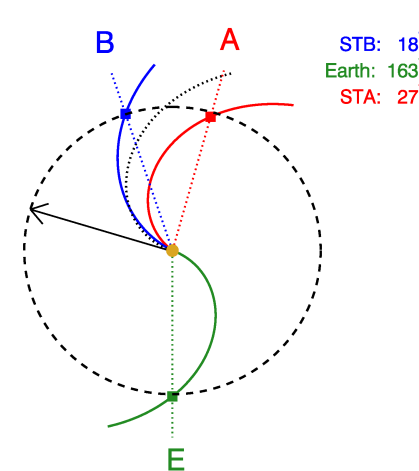

(b)
Fig. 11. a) STEREO-B EUVI $195 \AA$ image showing the jet/flare location in relation to the nominal magnetic footpoints of STA (red) and STB (blue) and the $\mathrm{CH}$ associated with the SEP events on 1 August 2014. b) The same as in Fig. 1.

We note that STB was longitudinally the closest s/c to the flare/CME origin, but radially it was the farthest one from the Sun, at a distance of 1.07 AU. STA was at a distance of $0.96 \mathrm{AU}$ and SOHO at 0.98 AU. From the shock arrival time and the s/c radial distances we estimate the averaged shock transit speed of 598, 597 and $653 \mathrm{~km} \mathrm{~s}^{-1}$ for STB, STA and SOHO, respectively. These speeds are significantly smaller than the CME speed of $1809 \mathrm{~km} \mathrm{~s}^{-1}$ close to the Sun suggesting a strong deceleration en route to $1 \mathrm{AU}$.

\subsection{The 1 August 2014 events}

Figure 10 presents two consecutive SEP events detected at STA and STB separated by seven hours. The time profiles of the two events are very similar at each s/c, but differ strongly from $\mathrm{s} / \mathrm{c}$ to $\mathrm{s} / \mathrm{c}$. The SEPs originated from two small homologous flares accompanied by recurrent long and narrow EUV jets appearing in the same AR at coordinates S22W55 as seen from STB (Fig. 11), and were slightly behind the west and east limb from STA and SOHO/Earth point of view, respectively. The

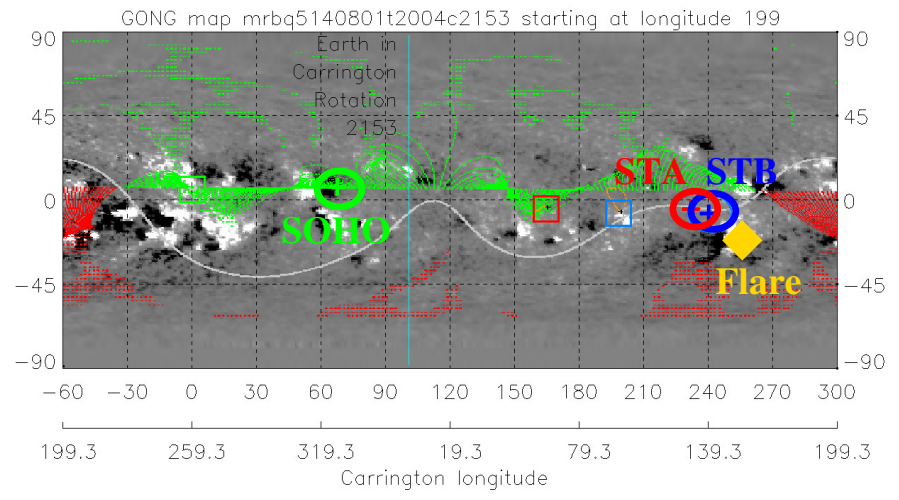

Fig. 12. Synoptic magnetic field map from GONG for events on $1 \mathrm{Au}-$ gust 2014. Description as in Fig. 3.

jets were about 1 solar radius long with prolongation into the coronograph (COR1) field of view (FOV) without any signatures in the COR2 FOV. Both SEP events were not associated with CMEs and EIT-waves or with type II radio bursts. They were accompanied merely by strong IP type III bursts as observed at STA/STB/WIND s/c, and the first one was also accompanied by a very weak coronal type III burst as observed by the ground based CALLISTO network (not shown). It should be mentioned, that the type III burst associated with the first event starting at 1622 UT (event I) was delayed relative to the jet appearance at 1613 UT by 9 min. At event II, the delay between the jet at 2316 UT and the type IIIs at 2324 UT was 8 min.

As shown in Fig. 10, STA measured two anisotropic impulsive events with sharp peaks and clear velocity dispersions (not shown). STB detected less impulsive profiles with poor velocity dispersions, smoothed maxima, and more prolonged decay phases. Although the first electrons reach the two s/c at the same pitch angle of $0^{\circ}$, in agreement with magnetic field polarity shown in Fig. 12, the PAD measured at STA is more anisotropic from the onset to the maximum, in contrast to STB.

Figure 12 presents the GONG synoptic magnetic field map showing the s/c backmapped magnetic footpoints and the location of the flare/jet associated with the SEP events. The angular separation between the flare/jet and the nominal footpoints of each s/c was relatively small, $27^{\circ}$ for STA and $18^{\circ}$ for STB, suggesting that the two s/c were similarly well connected to the parent source. At the same time, the magnetic footpoint of STB is slightly closer to the jet's location than STA. The exact actual location of the footpoints at the Sun's surface is generally difficult to estimate especially owing to the lack of knowledge of the magnetic field distribution below the source surface. Nevertheless, the relative positions of the flare, STB, and STA are aligned from southwest to northeast. The solar wind data and EUV observations confirm that this is also the ordering of the magnetic connections. Figure 13 presents the solar wind data and shows that on 1 August STB was situated inside a fast solar wind clearly streaming from the south $\mathrm{CH}$ (Fig. 11a). The same stream had already overtaken the STA s/c two to three days earlier, corresponding to the nominal longitudinal separation between STA and STB of $34^{\circ}$. The estimated nominal STB footpoint, shown in Figs. 11 and 12, is located outside the $\mathrm{CH}$. However, it is plausible to suggest that the actual STB footpoint is located farther southwards from the estimated location, i.e., close to the edge or inside of the south pole $\mathrm{CH}$ in agreement with the measured solar wind speed between 561-588 $\mathrm{km} \mathrm{s}^{-1}$ (Table 1). Such a shift could explain the observed high-speed solar wind stream flowing from the south $\mathrm{CH}$ (see Fig. 13). Consequently, we claim 
STEREO-A and STEREO-B Plasma and Magnetic Field Data

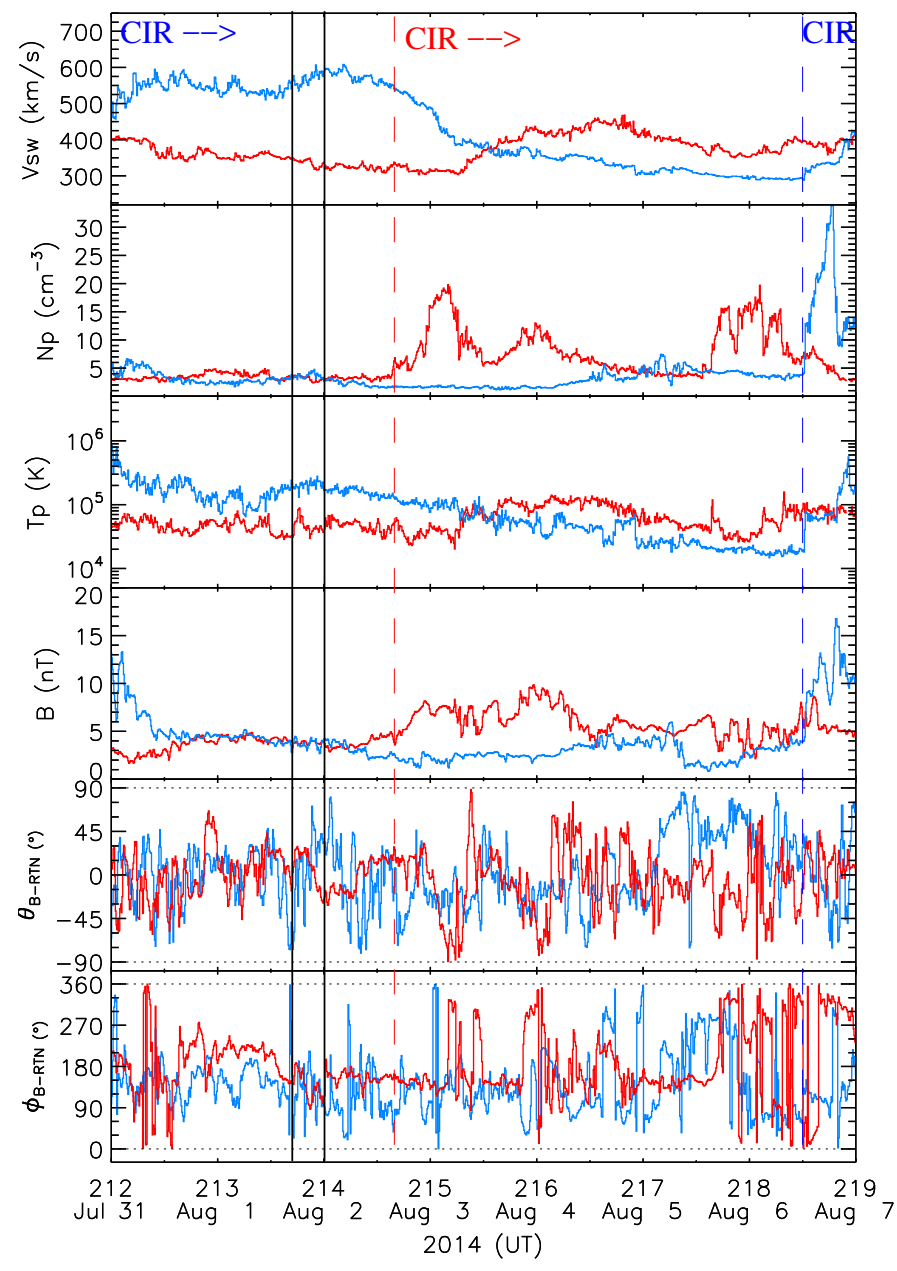

Fig. 13. Events on 1 August 2014. Solar wind and magnetic field data. SEP onset and CIRs are indicated by solid and dashed vertical lines, respectively.

that during both SEP events the real STA footpoint was at the same distance or even farther away from the parent source than the STB footpoint. Nevertheless, against the expectation, STA detected a higher electron peak intensity, an earlier and a more impulsive onset, and a higher anisotropy than STB.

On the one hand the strong difference in onset timing and intensity profiles for the s/c situated outside and inside of the $\mathrm{CH}$ might be explained by different propagation conditions along the paths towards STA and STB. On the other hand a mechanism shielding the penetration of electrons into $\mathrm{CH}$ regions and into high-speed stream could also cause the observations.

\subsection{Onset time delays: between STA and STB and relative to type III radio bursts}

To determine the onset time delay between the in situ measured nearly relativistic 55-65 keV electrons and type III radio bursts we used the solar release time. The inferred release time of electrons at the Sun is the measured onset time shifted back by the electron propagation time along the nominal Parker spiral whose length depends on the solar wind speed measured during the SEP onset. The release time of the type III burst at the Sun is the observed onset of the $16 \mathrm{MHz}$ emission at the s/c shifted back by the propagation time of electromagnetic emission from the
Sun to the s/c. We assume that the electron injection was coincident with decametric-hectometric (DH: 1-16 MHz) type III onsets (e.g., Kahler et al. 2007) as observed by SWAVES and that both the low-energy electrons responsible for type III generation and the nearly relativistic electrons belong to the same particle population.

Each of the four particle events detected at STA and at STB shows an onset delay relative to the associated type III burst onset and relative to each other as well. The onset delays between $\mathrm{s} / \mathrm{c}$ and type IIIs range between nearly 0 and $24 \mathrm{~min}$ and the onset delays between the two s/c range between 13 and $20 \mathrm{~min}$ (Table 1). At the same time the larger the delay between STA and STB, the larger the intensity difference between the two s/c.

The electron delays with respect to type IIIs imply that the effective propagation paths from the Sun to the s/c should be substantially larger than the path along the nominal Parker spiral of $\sim 1.2 \mathrm{AU}$ and range between 1.2-2.5 AU. These larger propagation paths are in agreement with the values found in previous investigations (e.g., Ragot 2006; Kahler et al. 2011; Klassen et al. 2015). Furthermore, the onset delays at STB relative to STA provide the difference in propagation path lengths between the two $\mathrm{s} / \mathrm{c}$ ranging between $\sim 0.7-1.0 \mathrm{AU}$, suggesting that the particles reach the two s/c along strongly different paths or propagation conditions. Figure 14a presents the SEP onset delays as a function of the magnetic footpoint separation for the four presented events and for an additional event on 2 May 2014 (Klassen et al. 2015). All five events in our sample show a behavior different from the expectation: the nominally best connected s/c always detected lower peak intensity and a larger onset delay than the nominally farther separated s/c (taking into account the two STEREO s/c only).

It should be noted that there are still alternative ideas that try to explain the delayed arrival of electrons because of delayed injection of nearly relativistic electrons relative to type III generating electrons (Krucker et al. 1999; Haggerty \& Roelof 2002; Klassen et al. 2002; Cane 2003; Wang et al. 2006).

\subsection{Angular and longitudinal intensity distribution: two- and three-spacecraft events}

Figure $14 \mathrm{~b}$ presents the peak intensity distribution relative to the angular separation between the s/c footpoints and the parent flare measured in the energy range of $55-65 \mathrm{keV}$. The peak intensities are normalized to the smallest peak for each event and the pre-event background is subtracted. As STB is normally 0.030.11 AU farther away from the Sun than STA the peak intensity at STB adjusted to 1 AU could be higher by 5-20\%. In addition to the four discussed events we added the SEP event observed on 2 May 2014 (Klassen et al. 2015), which also belongs to the category of events detected by closely spaced s/c. We note that, we take the angular separation instead of longitudinal separation used in previous studies (e.g., Lario et al. 2013; Dresing et al. 2014) because at relatively small footpoint separations between two s/c $\left(\leq 70^{\circ}\right)$, the angular separation reflects more precisely the actual angular distance between the s/c and the flare.

The presented distributions are strongly unexpected. We would expect a linear or an exponential decrease in the peak intensity with increasing separation to the parent source, assuming that the ejected particles build up an approximately symmetric distribution (e.g. Gaussian-like) around the optimal connecting field line to the source (e.g., Strauss \& Fichtner 2015). Instead, all the presented events show a deviating pattern. In events where the s/c separations to the flare differ, we find nearly identical intensities (SEPs on 25 February 2014 and 2 May 2014), and 


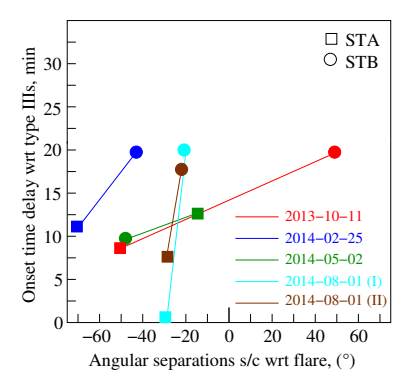

(a)

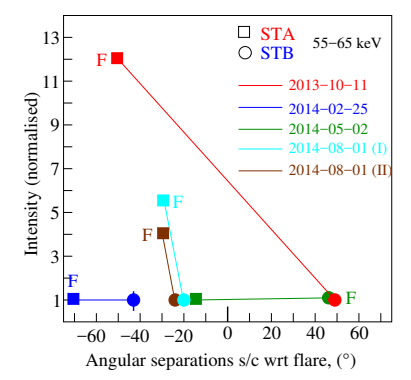

(b)
Fig. 14. a) Onset time delays of 55-65 keV electrons relative to the type III radio burst onset vs. angular separation between the parent flare and s/c footpoints as observed by STA ( $\square$ ) and STB (O). b) Angular distribution of peak electron intensities normalized to the smallest peak. The pre-event background is subtracted. The letter $(\mathrm{F})$ indicates the $\mathrm{s} / \mathrm{c}$ that detected the event onset first. The difference in radial distance to the Sun for each s/c is not taken into account; therefore, the values for STB could be 10-20\% higher. We note that the first two events (red and blue) are widespread and the third one (green) is a spike event.

in events with comparable s/c separations to the flare, we find strongly deviating intensities (remaining three events).

Furthermore, although both STA and STB footpoints were located to the east of the flare position during three events, one on 25 February 2014 with separation from the flare of $70^{\circ}$ and $42^{\circ}$, and events I/II on 1 August 2014 with separation of $27^{\circ} / 26^{\circ}$ and $18^{\circ} / 22^{\circ}$ for STA and STB, respectively, the electrons arrive first and peak earlier with comparable or higher peak intensities at the farthest one, STA s/c. We note that the STB peak intensity on 25 February 2014 event is not unambiguously determined because the intensity profile after the rising phase (Fig. 7) shows a flat-rising profile, which is probably caused by additional acceleration of the approaching IP shock. Therefore, the STB peak in Fig. $14 \mathrm{~b}$ is tagged with an error bar.

Figure 15 shows the typical presentation of the angular distribution of background-corrected peak intensities as measured at three locations around the Sun in comparable energy ranges at HET/STA and HET/STB (0.7-2.8 MeV), and EPHIN/SOHO at L1 (0.7-3.0 MeV). The observations are fitted by a symmetric Gaussian with a standard deviation of $\sigma=45^{\circ}$ and $\sigma=47^{\circ}$ for the widespread events on 11 October 2013 (a) and on 25 February 2014 (b), respectively. After pre-event background subtraction we applied a dividing factor of 13 to the $0.7-3.0 \mathrm{MeV}$ electron intensities measured by SOHO/EPHIN according to Lario et al. (2013). The strong difference in intensities measured by STA and STB during the event on 11 October 2013 when footpoints of the both s/c were approximately equally separated from the flare makes it practically impossible to provide a reasonable symmetric Gaussian fit centered on $0^{\circ}$. Indeed, the measured peak intensities imply a non-symmetric distribution in contrast to results found elsewhere (e.g., Dresing et al. 2014; Richardson et al. 2014; Strauss \& Fichtner 2015). The SEP on 25 February 2014 shows a different behavior. At higher energies of $0.7-3.0 \mathrm{MeV}$, the Gaussian fit reasonably matches the peak intensities; however, this is not the case for the lower energies of 55-65 keV (Fig. 14b) where the peak intensities at STA and STB are comparable. This indicates that the mechanism responsible for the longitudinal spreading of electrons accelerated in SEP events might be energy dependent.

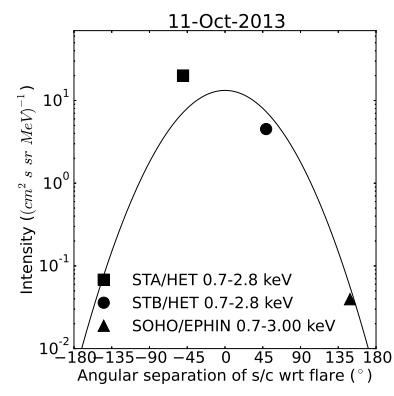

(a)

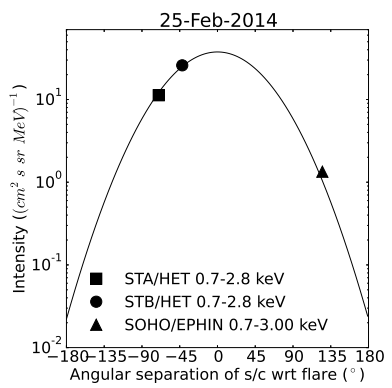

(b)
Fig. 15. Angular distribution and Gaussian fits to the peak intensities of

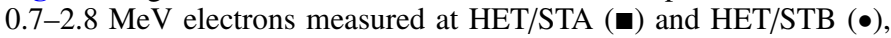
and 0.7-3.0 MeV electrons at EPHIN/SOHO ( $\boldsymbol{\Lambda}$ ) for the events on 11 October 2013 (a) and 25 February 2014 (b). Negative separation angles denote the s/c magnetic footpoint to the east from the flare. The preevent background has been subtracted.

\subsection{Observational results}

The studied SEP events were observed during relatively small longitudinal separations of $<72^{\circ}$ between the two STEREO s/c and during different spatial constellations of parent flare location with respect to nominal Parker spirals connecting the s/c to the Sun. The most important information of the events is summarized in Table 1. All four events show an unexpected intensity distribution and an unexpected relative onset timing at both $\mathrm{s} / \mathrm{c}$. Two studied events belong to so-called widespread SEP events and were additionally detected at $\mathrm{SOHO}$ at the L1 location. The observational results can be summarized as follows:

1. Generally for all four STEREO events the worst connected s/c (i.e., the farthest away) measured intensities that were similar to or higher than measured at the closest one, as shown in Fig. 14b. The strongest difference in peak intensities is of factor 12 as measured by STA and STB on 11 October 2013, although the footpoints of both s/c had the same angular separation to the flare.

2. All four events show a strongly unexpected relative onset timing at both STEREO s/c: The s/c nominally closest to the flare detected a later SEP onset than the farthest one with time delays up to $20 \mathrm{~min}$ (Fig. 14a and Table 1). Furthermore, in three events on 25 February 2014 and on 1 August 2014 (event I and II), although the footpoints of both STEREO s/c were located to the east of the flare position, the electrons arrive first and peak earlier at the farthest s/c (STA). All four events with higher peak intensity also show an earlier onset.

3. The effective length of the electron propagation paths from the Sun to both s/c reveals large differences up to $0.6 \mathrm{AU}$, although the orbital longitudinal separation between STA and STB was relatively small (between $72^{\circ}$ and $34^{\circ}$ ).

4. The widespread event on 11 October 2013 shows an asymmetric, non-Gaussian peak intensity distribution (Fig. 15).

\section{Discussion}

It has been shown for all presented SEP events that the angular peak intensity distribution at $1 \mathrm{AU}$ does not show the usual Gaussian distribution. This result together with the unexpected onset timing at both closely spaced STEREO s/c poses a challenge to each model and scenario suggested in previous studies (see e.g., the introduction in Gómez-Herrero et al. 2015). 


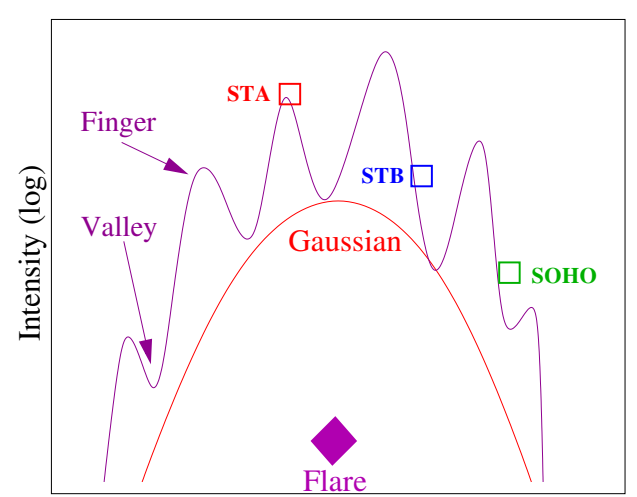

Angular separation, s/c wrt flare

Fig. 16. Proposed spatial peak intensity distribution of SEP events at $1 \mathrm{AU}$ vs. angular separation of the $\mathrm{s} / \mathrm{c}$ with respect to the flare.

Therefore, we propose a scenario containing a Gaussian-like intensity distribution (approximately uniform) superimposed by fringes or fingers. Figure 16 shows an intensity contour plot explaining the idea. In contrast to a simple Gaussian distribution such a combined distribution could explain the observed peak intensities and onset delays and may be valid not only for SEP events with relatively small angular extent but also on larger angular scales during widespread or circumsolar events. Depending on the location of each individual $\mathrm{s} / \mathrm{c}$ relative to the fingers, i.e. at the fingertip or between them, the measured intensity could be very different even for closely spaced s/c with a relatively small separation from each other.

The origin of fingers and valleys is not clear yet; they might be caused by different phenomena in the corona, possibly occurring mainly below the source surface. We propose that the open magnetic fields from the flaring region prolonged into the IP medium provide a prompt access of energetic particles into the IP medium, and form the fingers. Consequently, the s/c situated on the fingers detect not only a higher intensity, but also an earlier SEP onset. The valleys between the fingers could be formed by large-scale "closed" magnetic fields around the ARs, and/or by the $\mathrm{CHs}$ and the high-speed streams with a stronger turbulence and consequently with a higher scattering (e.g., Fig. 11 in Dresing et al. 2014; Crooker et al. 1999). In this case the $\mathrm{CHs}$, the high-speed streams and the stream interfaces could play the role of diffusion barriers, inhibiting the perpendicular diffusion and partially shielding the penetration of particles inside these regions.

Additionally, there are also other scenarios mentioned in previous studies (e.g., Kallenrode 1993; Dresing et al. 2012, 2014; Lario et al. 2014; Klassen et al. 2015) that could be responsible for the finger-valley distribution:

1. a strong deviation of the interplanetary magnetic field from the nominal Parker spiral. For example, an en route CME/ICME may significantly displace the optimal magnetic connection to the source towards the s/c detecting the highest intensity. This implies that the normally used backmapping method for the magnetic field connecting the s/c to the Sun, i.e. the nominal Parker spiral, is not valid at least for the presented events observed by closely spaced s/c;

2. a fan-shaped, strongly tilted and non-radial magnetic field connecting the parent flare/source to the source surface (Klein et al. 2008);

3. a large-scale meandering field lines, which can lead to the mixing of empty magnetic flux tubes and those filled with energetic particles due to some flux tubes being connected to the source region and others not. This can result in very small local cross-field diffusion as observed for instance during so-called "dropout" events (e.g., Mazur et al. 2000; Giacalone \& Jokipii 2012; Laitinen et al. 2013) and may form a finger intensity distribution;

4. strongly differing IP propagation conditions (e.g., Marsch 2006; Ragot 2006) along the different paths to each s/c, which may essentially affect the electron mean free path, the anisotropy, the intensity and consequently the onset time at $1 \mathrm{AU}$;

5. a global asymmetric CME/shock, which determines the SEP injection and the intensity distribution at different longitudes (Lario et al. 2016);

6. multi-point injections into different coronal magnetic structures below the source surface, as suggested by Wang et al. (2014) and Klassen et al. (2015).

Different sophisticated scenarios involving a combination of the above-mentioned mechanisms may also come into consideration when explaining of the unexpected distribution of observed individual events.

The suggested scenario where the open field lines of the flaring AR provide prompt access to the IP medium forms the fingers and where the $\mathrm{CH}$ and its associated high-speed stream forms the valleys seems to be more favorable in relation to the presented SEP events. Indeed, during the three events on 25 February 2014 and on 1 August 2014 - when the STB footpoints were situated inside or on the outskirts of $\mathrm{CHs}$ and when the s/c itself was inside the CIRs (Figs. 5 and 10a) - it measured less intense and less impulsive time profiles, and delayed onsets with respect to the STA situated outside the high-speed stream. The magnetic connection to the $\mathrm{CH}$ is confirmed by the observation of high-speed solar wind streams measured at STB at that time (Figs. 9 and 13). Obviously, different levels of turbulence in slow- and high-speed solar wind streams imply different values of perpendicular transport/diffusion and of the propagation mean free path. Therefore, a higher turbulence and scattering inside the $\mathrm{CHs}$ and CIRs may partly shield the penetration of particles, reduce the peak intensity, and delay the particle arrival at the s/c situated inside a high-speed stream. Strong differences in electron intensities were also observed at Ulysses when it was situated in high-speed solar wind $\mathrm{CH}$ streams at high heliographic latitudes in comparison to $1 \mathrm{AU}$ measurements in the ecliptic at the ACE s/c (Lario et al. 2003). In this case, a strong intensity reduction inside the $\mathrm{CHs}$ and a non-Gaussian SEP distribution at $1 \mathrm{AU}$ contradicts the idea of a strong and fast perpendicular particle spread in the corona and in the IP medium as suggested by Dresing et al. (2012) and Dröge et al. (2014) for widespread SEP events. In addition, we cannot exclude an additional unknown mechanism preventing particle penetration into the $\mathrm{CH}$ through its border.

For the SEP event on 11 October 2013 the situation is less obvious and a combination of scenarios 2, 3, 5 may be responsible for the observed timing and intensity distribution because both STEREO s/c were situated inside a slow solar wind during relatively quiet and similar IP conditions. Therefore a nonradial electron injection pattern could play an important role in building up the unexpected intensity distribution. Such nonradial electron injection is often observed by tracing of type III radio bursts showing non-radial trajectories and positions relative to the parent flare (e.g., Klassen et al. 1999, 2012). The same is valid for type II radio bursts and coronal shocks, which sometimes show a non-radial/azimuthal propagation trajectory in the corona (Aurass et al. 1998). 
The observed unexpected angular intensity distribution at $1 \mathrm{AU}$ and proposed finger distribution can be also interpreted as a two-component particle distribution (Klassen et al. 2005; Kocharov et al. 2015). The first one is a prompt component (fingers) implying a relatively narrow injection pattern because of direct particle injection along the open field lines. The second one (Gaussian-like) implies a prolonged-in-time and broadin-space component, which may originate from trapped particles undergoing cross-field diffusion in the corona and IP medium. Such a trapped population of relativistic electrons was already observed during so-called radio-CMEs (Bastian et al. 2001). These electrons could be trapped for a longer time and escape into IP space owing different processes (e.g., local reconnections, diffusion, etc.) and may explain the broadness of the SEP distribution around the Sun for widespread SEP events and a relative small difference in electron intensities measured at STEREO and at SOHO, although their angular separation to the source was relatively large $\left(70^{\circ}\right.$ and $\left.125^{\circ}\right)$ as in the SEP event on 25 February 2014. Alternatively, a shock propagating towards the s/c which continues to accelerate particles could also be the reason for a broad Gaussian-like distribution as suggested by Lario et al. (2016).

\section{Summary}

We presented a study of four solar electron events observed by the two closely spaced STEREO s/c during the approach to the superior solar conjunction between October 2013 and August 2014, and by SOHO at L1. During this period the longitudinal separation between STA and STB was relatively small, between $72^{\circ}$ and $34^{\circ}$.

All presented events show an unexpected peak intensity distribution, and an unexpected SEP onset timing between the two STEREO s/c and also with respect to the associated type III radio bursts. The strong difference in peak intensities (a factor of $\leq 12$ ) and the delayed particle onset at the s/c nominally closest connected to the flare in comparison to the farther one led us to suggest a concept of rippled peak intensity distribution (i.e., quasi uniform Gaussian plus fingers) at $1 \mathrm{AU}$.

The fingers could be formed by the open field lines connecting the parent source to the s/c through the source surface allowing a fast particle transport from the source to the $\mathrm{s} / \mathrm{c}$. The valleys between the fingers could be formed by $\mathrm{CHs}$ and their associated high-speed streams, shielding partially the penetration of particles inside of it and preventing a prompt particle propagation. This effect could delay the particle arrival times at $1 \mathrm{AU}$ and reduce the observed intensities. This concept in combination with non-radial particle injection along the non-radial magnetic field distribution below the source surface may explain the unusual particle distributions and onset timing in the presented SEP events.

Acknowledgements. We thank the STEREO SWAVES, IMPACT, SECCHI, PLASTIC, MAG and GONG teams for providing the data used in this paper and the FESTIVAL team who developed the browser for solar imaging data. The authors thank the anonymous referee for valuable comments. R. Gómez-Herrero acknowledges the financial support by the Spanish Ministerio de Economía y
Competitividad under project ESP2013-48346-C2-1-R and the University of Alcalá under project CCG2015/EXP-055. The STEREO/SEPT and SOHO/EPHIN projects are supported under grant 50 OC 1302 by the German Bundesministerium für Wirtschaft through the Deutsches Zentrum für Luft- und Raumfahrt (DLR).

\section{References}

Acuña, M. H., Curtis, D., Scheifele, J. L., et al. 2008, Space Sci. Rev., 136, 203 Aurass, H., Hofmann, A., \& Urbarz, H.-W. 1998, A\&A, 334, 289

Bastian, T. S., Pick, M., Kerdraon, A., Maia, D., \& Vourlidas, A. 2001, ApJ, 558, L65

Bougeret, J. L., Goetz, K., Kaiser, M. L., et al. 2008, Space Sci. Rev., 136, 487 Cane, H. V. 1996, in AIP Conf. Proc., 374, 124

Cane, H. V. 2003, ApJ, 598, 1403

Crooker, N. U., Gosling, J. T., Bothmer, V., et al. 1999, Space Sci. Rev., 89, 179 Dresing, N., Gómez-Herrero, R., Klassen, A., et al. 2012, Sol. Phys., 281, 281 Dresing, N., Gómez-Herrero, R., Heber, B., et al. 2014, A\&A, 567, A27

Dröge, W., Kartavykh, Y. Y., Klecker, B., \& Kovaltsov, G. A. 2010, ApJ, 709, 912

Dröge, W., Kartavykh, Y. Y., Dresing, N., Heber, B., \& Klassen, A. 2014, J. Geophys. Res. (Space Phys.), 119, 6074

Galvin, A. B., Kistler, L. M., Popecki, M. A., et al. 2008, Space Sci. Rev., 136, 437

Giacalone, J. \& Jokipii, J. R. 2012, ApJ, 751, L33

Gómez-Herrero, R., Dresing, N., Klassen, A., et al. 2015, ApJ, 799, 55

Haggerty, D. K., \& Roelof, E. C. 2002, ApJ, 579, 841

Howard, R. A., Moses, J. D., Vourlidas, A., et al. 2008, Space Sci. Rev., 136, 67

Huttunen-Heikinmaa, K., Valtonen, E., \& Laitinen, T. 2005, A\&A, 442, 673

Kahler, S. W., Aurass, H., Mann, G., \& Klassen, A. 2007, ApJ, 656, 567

Kahler, S. W., Haggerty, D. K., \& Richardson, I. G. 2011, ApJ, 736, 106

Kallenrode, M.-B. 1993, J. Geophys. Res., 98, 5573

Klassen, A., Aurass, H., Klein, K.-L., Hofmann, A., \& Mann, G. 1999, A\&A, 343,287

Klassen, A., Bothmer, V., Mann, G., et al. 2002, A\&A, 385, 1078

Klassen, A., Krucker, S., Kunow, H., et al. 2005, J. Geophys. Res. (Space Phys.), 110, A09S04

Klassen, A., Gómez-Herrero, R., Heber, B., et al. 2012, A\&A, 542, A28

Klassen, A., Dresing, N., Gómez-Herrero, R., \& Heber, B. 2015, A\&A, 580, A115

Klein, K.-L., Krucker, S., Lointier, G., \& Kerdraon, A. 2008, A\&A, 486, 589

Kocharov, L., Klassen, A., Valtonen, E., Usoskin, I., \& Ryan, J. M. 2015, ApJ, 811, L9

Krucker, S., Larson, D. E., Lin, R. P., \& Thompson, B. J. 1999, ApJ, 519, 864

Laitinen, T., Dalla, S., \& Marsh, M. S. 2013, ApJ, 773, L29

Lario, D., Roelof, E. C., Decker, R. B., \& Reisenfeld, D. B. 2003, Adv. Space Res., 32, 579

Lario, D., Aran, A., Gómez-Herrero, R., et al. 2013, ApJ, 767, 41

Lario, D., Raouafi, N. E., Kwon, R.-Y., et al. 2014, ApJ, 797, 8

Lario, D., Kwon, R.-Y., Vourlidas, A., et al. 2016, ApJ, 819, 72

Marsch, E. 2006, Liv. Rev. Sol. Phys., 3, 1

Mazur, J. E., Mason, G. M., Dwyer, J. R., et al. 2000, ApJ, 532, L79

Müller-Mellin, R., Kunow, H., Fleissner, V., et al. 1995, Sol. Phys., 162, 483

Müller-Mellin, R., Böttcher, S., Falenski, J., et al. 2008, Space Sci. Rev., 136, 363

Ragot, B. R. 2006, ApJ, 653, 1493

Richardson, I. G., von Rosenvinge, T. T., Cane, H. V., et al. 2014, Sol. Phys., 289, 3059

Strauss, R. D., \& Fichtner, H. 2015, ApJ, 801, 29

von Rosenvinge, T. T., Reames, D. V., Baker, R., et al. 2008, Space Sci. Rev., 136,391

Wang, L., Lin, R. P., Krucker, S., \& Gosling, J. T. 2006, Geophys. Res. Lett., 33, 3106

Wang, Y., Qin, G., Zhang, M., \& Dalla, S. 2014, ApJ, 789, 157

Wibberenz, G., \& Cane, H. V. 2006, ApJ, 650, 1199

Wiedenbeck, M. E., Mason, G. M., Cohen, C. M. S., et al. 2013, ApJ, 762, 54 


\section{Appendix A: Additional table}

Table A.1. Event properties.

\begin{tabular}{|c|c|c|c|c|}
\hline Properties/Event & 2013-Oct-11 & 2014-Feb-25 & 2014-Aug-01 (I) & 2014-Aug-01 (II) \\
\hline \multicolumn{5}{|l|}{ Electron onset time, UT: } \\
\hline - STA & $0734^{a}$ & $0109^{a}$ & 1634 & 2345 \\
\hline$-\mathrm{STB}$ & $0747^{a}$ & $0122^{a}$ & 1654 & 2354 \\
\hline$-\mathrm{SOHO}^{d}$ & $>1110$ & $\leq 0204$ & - & - \\
\hline Delay STB vs. STA, min & 13 & 13 & 20 & 9 \\
\hline \multicolumn{5}{|l|}{ Peak intensity ${ }^{b},\left(\mathrm{~cm}^{2} \text { sr MeV }\right)^{-1}$ : } \\
\hline - STA & $1.35 \times 10^{5}$ & $7.6 \times 10^{4}$ & 4800 & 9200 \\
\hline$-\mathrm{STB}$ & $1.16 \times 10^{4}$ & $>4.3 \times 10^{4}<10.3 \times 10^{4}$ & 870 & 2300 \\
\hline$-\mathrm{SOHO}^{d}$ & 65 & 3600 & - & - \\
\hline Peak intensity ratio, STA/STB & 11.6 & $1.8-0.74$ & 5.5 & 4.0 \\
\hline Peak spectral index ${ }^{e}, \gamma$, STA/STB & $1.9 / 1.5$ & $2.0 / 1.6$ & $3.3 / 2.7$ & $3.0 / 2.6$ \\
\hline \multicolumn{5}{|l|}{ Mag. footpoints separation, ${ }^{\circ}$ : } \\
\hline - STA - flare & 50 & 70 & 27 & 26 \\
\hline - STB - flare & 49 & 42 & 18 & 22 \\
\hline - SOHO - flare & 149 & 125 & 163 & 158 \\
\hline Flare/Jet onset, UT & $\leq 0710.5$ & $\leq 0043$ & $1613^{\mathrm{SDO}}$ & $\leq 2316^{\text {STA, SDO }}$ \\
\hline Type III burst onset (Waves), UT & 0712 & 0046 & 1622 & 2324 \\
\hline \multicolumn{5}{|l|}{ Delay, electrons vs. type III, min: } \\
\hline - STA & 8.2 & 10.0 & -0.6 & 27.1 \\
\hline$-\mathrm{STB}$ & 18.5 & 23.8 & 20.0 & 18.1 \\
\hline$-\mathrm{SOHO}^{d}$ & $>238$ & 78 & - & - \\
\hline Flare Carr. Long., ${ }^{\circ}$ & 83 & 108 & 155 & 155 \\
\hline Flare Carr. Lat., & 21 & -13 & -22 & -22 \\
\hline \multicolumn{5}{|l|}{$V_{\mathrm{sw}}, \mathrm{km} \mathrm{s}^{-1}$} \\
\hline - STA & 351 & 372 & 349 & 328 \\
\hline$-\mathrm{STB}$ & 321 & 564 & 557 & 584 \\
\hline$-\mathrm{SOHO}$ & 397 & 464 & 340 & 403 \\
\hline Type II burst onset, UT & $0711^{c}$ & $0045.5^{c}$ & No & No \\
\hline CME, speed, $\mathrm{km} \mathrm{s}^{-1}$; angular width, ${ }^{\circ}$ & 1163/halo & 1809/halo & No & No \\
\hline EIT-wave & yes & yes & No & No \\
\hline Local Langmuir waves & STA & STB? & No & No \\
\hline Distance STA-Sun, AU & 0.97 & 0.96 & 0.97 & 0.97 \\
\hline Distance STB-Sun, AU & 1.06 & 1.07 & 1.00 & 1.00 \\
\hline Long. separation STA vs. STB, ${ }^{\circ}$ & 72 & 47 & 34 & 34 \\
\hline
\end{tabular}

Notes. ${ }^{(a)}$ Onset determined using the Poisson-CUSUM method (Huttunen-Heikinmaa et al. 2005), remainders by n-sigma threshold approach (e.g. Krucker et al. 1999). ${ }^{(b)} 5$ min averaging and the background is subtracted. ${ }^{(c)}$ Ground based observations. ${ }^{(d)} 250-700 \mathrm{keV}$ (EPHIN/SOHO instrument). ${ }^{(e)}$ Derived power-law index from the omnidirectional peak intensity spectrum in the energy range 55-335 keV. Flare latitude (+) means north, solar wind speed $\left(V_{\mathrm{sw}}\right)-1 \mathrm{~h}$ average data taken during the SEP onset. All parameters are derived for 55-65 keV electrons after background subtraction. 